\title{
Robots en la educación de la primera infancia: aprender a secuenciar acciones usando robots programables
}

\section{(Robots in the Early Childhood Education: learning to sequence actions using programmable robots)}

\author{
Yen-Air Caballero-González \\ Ana García-Valcárcel Muñoz-Repiso \\ Universidad de Salamanca, USAL (España)
}

DOI: http://dx.doi.org/10.5944/ried.24.1.27508

\section{Cómo referenciar este artículo:}

Caballero-González, Y., y García-Valcárcel Muñoz-Repiso, A. (2021). Robots en la educación de la primera infancia: aprender a secuenciar acciones usando robots programables. RIED. Revista Iberoamericana de Educación a Distancia, 24(1), pp. 77-94. doi: http://dx.doi.org/10.5944/ried.24.1.27508

\section{Resumen}

El incremento y avance tecnológico que experimenta la sociedad actualmente está impulsando el desarrollo de iniciativas educativas que integran actividades de enseñanzaaprendizaje para fomentar habilidades digitales asociadas a la programación y el pensamiento computacional como es la secuenciación de movimientos. En este trabajo se muestran algunos de los resultados que se obtuvieron con la realización de una experiencia de aprendizaje sobre el pensamiento computacional orientado a los niños en la educación temprana. En las actividades se efectuaron retos de programación utilizando el robot Bee-Botß. El estudio contempló evaluaciones de tipo pretest/postest utilizando grupo control. La muestra de participantes fue de 40 estudiantes pertenecientes a un centro educativo, ubicado en Salamanca, España. Las actividades se desarrollaron en el curso 2018-2019. La recolección de datos sobre el dominio alcanzado por los estudiantes, en las evaluaciones desarrolladas, se efectuó mediante el uso de una rúbrica de evaluación. Los datos recolectados nos permitieron conocer la existencia de diferencias significativas en la capacidad de secuenciación de acciones a favor de los estudiantes que participaron de las actividades de aprendizaje con robots. Por otro lado, se pudo afirmar que no se encontraron diferencias asociadas al sexo de los participantes en relación con la programación y construcción de secuencias.

Palabras clave: robótica; programación; aprendizaje; pensamiento crítico; educación de la primera infancia. 


\begin{abstract}
The increase and technological advance that society is currently experiencing is driving the development of educational initiatives that integrate teaching-learning activities to foster digital skills associated with programming and computational thinking such as movement sequencing. In this paper, we show some of the results that were obtained with the realization of a learning experience on computational thinking oriented to children in early education. The activities included programming challenges using the Bee-Bot $®$ robot. The study included pre-test/post-test evaluations using a control group. The sample of participants was 40 students from an educational centre, located in Salamanca, Spain. The activities were developed in the academic year 2018-2019. The collection of data on the mastery reached by the students, in the developed evaluations, was carried out using an evaluation rubric. The collected data allowed us to know the existence of significant differences in the capacity of the sequencing of actions in favour of the students who participated in the learning activities with robots. On the other hand, it was possible to affirm that no differences were found associated with the sex of the participants in relation to the programming and construction of sequences.
\end{abstract}

Keywords: robotics; programming; learning; critical thinking; early childhood education.

El avance generado por la integración de las Tecnologías de Información y Comunicación (TIC) en los diferentes sectores de actividad y participación social ha motivado que los sistemas educativos ofrezcan la posibilidad de fomentar habilidades y competencias necesarias para enfrentar los retos que impone este nuevo ecosistema social (García-Valcárcel y Hernández, 2013; Henriksen et al., 2018). En este nuevo contexto de acción y participación la tecnología y la innovación son factores determinantes del progreso y el bienestar, promoviendo el diseño de escenarios para la enseñanza-aprendizaje más diversos, flexibles y participativos centrados en el estudiante como elemento clave del proceso (Berrocoso, Sánchez y Arroyo, 2015).

Por otro lado, la combinación de recursos de aprendizaje basados en la tecnología y la aplicación de técnicas y métodos activos son iniciativas que han irrumpido con gran fuerza en los diferentes niveles y contextos de aprendizaje (formal e informal). Uno de los focos de interés consiste en lograr el desarrollo de experiencias pedagógicas activas permitiendo la adquisición de nuevas alfabetizaciones, como la código-alfabetización, el pensamiento computacional, aprendizajes en las áreas STEM (por los términos en inglés Science, Technology, Engineering and Math) y otras habilidades de tipo social entre los participantes (Cejka, Rogers y Portsmore, 2006; Bers et al., 2014; González y García-Valcárcel, 2017; Strawhacker y Bers, 2019).

La código-alfabetización, se refiere a las nuevas teorías y prácticas que facilitan que una persona pueda comunicarse efectivamente con diversos dispositivos tecnológicos empleando instrucciones escritas en lenguajes informáticos (Vee, 2013; Liu, Perera, 
y Klein, 2017). Este proceso conlleva el dominio cognitivo de una nueva forma de pensar, denominada pensamiento computacional (Papert, 1980; Wing, 2006). En los últimos años existe un gran interés por apoyar el diseño e implementación de iniciativas y propuestas de enseñanza-aprendizaje del Pensamiento Computacional (PC) orientados a contextos de aprendizaje de primeros niveles educativos (ZapataRos, 2015; García-Peñalvo y Mendes, 2018).

Las prácticas pedagógicas que se han planteado para el desarrollo del pensamiento computacional desde la infancia temprana se fundamentan en las teorías y enfoques de aprendizaje constructivistas y construccionistas propuestas por Jean Piaget y Seymour Papert respectivamente (Lee, Martín y Apone, 2014; Sullivan y Bers, 2018). En ambas corrientes la figura central del proceso enseñanza-aprendizaje es el estudiante.

Sin embargo, fue Seymour Papert quien otorga el carácter singular y la importancia al desarrollo de estrategias educativas vinculadas al aprendizaje de la informática, la programación y la computación desde la infancia temprana (Bers, 2008; Kucuk y Sisman, 2017; González y García-Valcárcel, 2018). En este sentido, Papert introduce la robótica como un recurso de tecnología educativa que posee las propiedades y características adecuadas para alcanzar un desarrollo significativo de las nuevas habilidades tecnológicas y sociales (Bers, 2017; Bruni y Nisdeo, 2017).

La Robótica Educativa (RE) aporta un significado lúdico y motivacional al proceso enseñanza-aprendizaje a través de la utilización de principios pedagógicos fundamentados en la interactividad y el desarrollo de actitudes positivas como el trabajo colaborativo, la creatividad, la comunicación y el liderazgo (Resnick y Rosenbaum, 2013; González-Martínez, Estebanell-Minguell y Peracaula-Bosch, 2018).

La robótica como disciplina de conocimiento busca estimular el interés de los estudiantes generando nuevas formas de pensamiento que facilitan de manera gradual la estructuración del pensamiento lógico y formal utilizando para ello: retos, proyectos y experimentos vinculados al entorno y la realidad que caracteriza el medio social donde el participante desarrolla sus actividades cotidianas (Kalelioğlu, 2015).

En trabajos previos se ha establecido el potencial de la RE para desarrollar estrategias de aprendizaje que promuevan la adquisición de habilidades y conocimientos en campos que tradicionalmente están orientados al género masculino (Cheng, Sun y Chen, 2018; González-González, 2019). Igualmente, se exponen las posibilidades que existen al realizar propuestas de aprendizaje en etapas educativas tempranas, donde los estereotipos de género aún no se han consolidado de forma permanente (Metz, 2007).

Sin embargo, se informa que un número significativo de propuestas formativas basadas en el dominio y aprendizaje de la tecnología, en niñas, se focaliza hacia los niveles educativos medios desaprovechando, con esto, las ventajas pedagógicas propias de los primeros años de educación formal (Sullivan y Bers, 2013). 
Además, en relación con el desarrollo del PC se describen en la literatura una serie de conceptos, dimensiones o características que son utilizadas como marco de referencia para el diseño e implementación de estrategias educativas asociadas al desarrollo de esta forma de pensamiento propia de una sociedad de la información y del conocimiento.

En este sentido, la secuenciación o construcción de secuencias, es una de las habilidades asociadas al PC que se vincula tanto a los programas curriculares como a las evaluaciones de aprendizaje de estudiantes de niveles educativos iniciales (Strawhacker y Bers, 2015; Relkin y Bers, 2019). Actualmente, la evaluación del pensamiento computacional y de esta característica, en niños pequeños, es posible gracias a recursos y materiales de tipo tangible como los robots programables (González-González, 2019; Siu-Cheung, 2019). Sin embargo, recientemente surge el concepto de pensamiento computacional desenchufado que promueve el uso de otro tipo de recursos para la evaluación del desempeño en esta área de conocimiento sin el uso de ordenadores, robots u otro tipo de dispositivos (Zapata-Ros, 2019).

Desde el punto de vista conceptual, una de las primeras aproximaciones las presenta en el 2006, Jeannette Wing, su argumento sostiene que el PC "representa un conjunto de habilidades y actitudes, aplicable universalmente; que toda persona (no sólo los científicos de la computación) deberían adquirir y utilizar" (Wing, 2006, p. 33).

En 2009 la Computer Science Teachers Association y la International Society for Technology in Education desarrollaron una definición operativa sobre PC describiéndolo como un proceso de resolución de problemas que incluye características como la formulación de problemas, organización y análisis de datos de manera lógica, abstracción, pensamiento algorítmico y generalización de soluciones (Ramírez y Sosa, 2013; Sullivan y Bers, 2016).

Otra iniciativa conceptual sobre PC con gran impacto en el escenario internacional fue la propuesta denominada «computational thinking framework». En ella se estructura el aprendizaje del PC en base a tres dimensiones: los conceptos, las prácticas y las perspectivas (Brennan y Resnick, 2012).

Grover y Pea (2013) orientan el desarrollo PC en niveles K12 a través de características que proporcionan aceptación y confiabilidad, entre las que están: la abstracción, generalización de patrones, procesamiento sistemático de la información, sistemas de símbolos y representación, noción algorítmica de control de flujo, descomposición estructurada de problemas o modularización, pensamiento iterativo, recursivo y paralelo así como la lógica condicional, la depuración y detección sistemática de errores.

En este mismo año Selby y Woollard (2013) definen el PC como un proceso cognitivo o mental, de los humanos, no de las máquinas involucrando habilidades como la abstracción, descomposición, pensamiento algorítmico, evaluación y generalización. En otros estudios y análisis de la literatura científica sobre PC se realiza un énfasis hacia el dominio de características como la construcción y diseño 
de algoritmos, incluyendo la comprensión de la secuenciación de acciones y el flujo de control (Angeli et al., 2016; Papadakis, Kalogiannakis y Zaranis, 2016).

Por otro lado, Bers y su equipo de investigación han realizado varios aportes de tipo experimental donde se argumentan las posibilidades y beneficios que se pueden obtener al introducir el aprendizaje del PC en niños de la infancia temprana. Se exponen el desarrollo del PC a través del dominio de 7 poderosas ideas como los algoritmos, modularidad, las estructuras de control, representación, hardware/ software, proceso de diseño y depuración (Bers, 2008; Bers, 2010; Bers, 2012; Bers, 2018).

Igualmente, para fortalecer el desarrollo de competencias y habilidades digitales orientado a contextos educativos de los primeros años se han propuesto iniciativas con un diseño instruccional basado en actividades y recursos sencillos o desenchufados (Zapata-Ros, 2019).

En estas iniciativas se integran tareas como el dibujo, la construcción, realización de competiciones, juegos de mesa y el uso de kits de robótica. Además, se argumenta que las actividades que se planifiquen deberán orientarse utilizando los postulados expuestos en teorías del aprendizaje cognitivista clásicas (Merrill, 2009; Reigeluth, 2016).

En el presente trabajo de investigación se pretende saber si las actividades de robótica que realizan los niños y niñas de educación infantil desarrollan su capacidad de secuenciación y se exponen algunos resultados logrados mediante el desarrollo de una experiencia de aprendizaje sobre habilidades digitales de pensamiento computacional y programación, en etapas educativas tempranas. La experiencia se organizó mediante retos de programación con resolución de problemas utilizando robots educativos programables (Caballero-González y García-Valcárcel, 2019; González y García-Valcárcel, 2020).

\section{MÉTODOLOGÍA}

Se tomó como referencia principal los resultados que fueron obtenidos mediante el desarrollo de una experiencia de formación y aprendizaje sobre pensamiento computacional. Las actividades se orientaron a estudiantes de educación infantil que pertenecían a un centro educativo concertado, ubicado en Salamanca, España. La experiencia se planificó y ejecutó utilizando un enfoque metodológico cuantitativo, mediante un diseño cuasiexperimental. Se utilizan dos grupos del mismo nivel (educación infantil) uno será el grupo control (no participará de las actividades formativas) y otro el grupo experimental (se expondrá a las actividades de aprendizaje). Los datos sobre el dominio y aprendizaje alcanzado por los estudiantes fueron recolectados aplicando evaluaciones del tipo pretest/postest con grupo control (Campbell y Stanley, 1993; Hernández et al., 2014). 


\section{Objetivo del estudio}

El estudio se desarrolló con el propósito de conocer cómo influyen las actividades de aprendizaje propuestas en la experiencia educativa orientadas al aprendizaje del pensamiento computacional orientada a estudiantes de primeros niveles escolares.

Se estableció como hipótesis de partida que los estudiantes que participan en las actividades propuestas alcanzan un aprendizaje del pensamiento computacional en relación con el dominio de la característica secuencia. En este sentido, mediante el desarrollo de las diferentes tareas que formaron la experiencia educativa se buscó dar respuesta fundamentalmente a dos interrogantes:

1. ¿Es posible que los estudiantes de primeros niveles educativos puedan adquirir un dominio en la construcción de secuencias y el pensamiento computacional al participar en una experiencia de formación y aprendizaje mediante retos con robots programables?

2. ¿Existe alguna diferencia asociada al sexo de los participantes en relación con el dominio de la característica secuencia del pensamiento computacional alcanzado por los estudiantes de niveles educativos iniciales que participan en una actividad de aprendizaje?

\section{Participantes (muestra)}

En las actividades propuestas como parte de la experiencia educativa participaron un total de 40 estudiantes que pertenecen al segundo ciclo de educación infantil, de un colegio concertado, ubicado en Salamanca, España. Las actividades se realizaron durante el segundo trimestre del curso académico 2018-2019. Para la experiencia se organizaron los estudiantes en dos grupos: experimental y control. Cada grupo lo integran 20 estudiantes que representan clases completas. Los estudiantes fueron distribuidos en cada clase de forma previa al desarrollo de la experiencia y por criterios propios del centro educativo y ajenos al estudio.

El propósito de la experiencia fue notificado a los padres de familia de los estudiantes mediante las vías de comunicación que habitualmente se utilizan en el centro educativo. Los estudiantes que conforman la muestra no habían participado en actividades de formación y aprendizaje previas a la realización del estudio.

La distribución por sexo de los participantes en ambos grupos (experimental y control) fue de 11 niños (55\% de los estudiantes) y 9 niñas ( $45 \%$ de los estudiantes). La edad de los participantes estuvo en el rango de 3 a 4 años.

\section{Procedimiento utilizado}

La experiencia formativa y de aprendizaje realizada implicó un total de 24 horas. En la figura 1 se muestra la estructura de actividades que la conforman. 
Figura 1. Actividades que componen experiencia de formación y aprendizaje

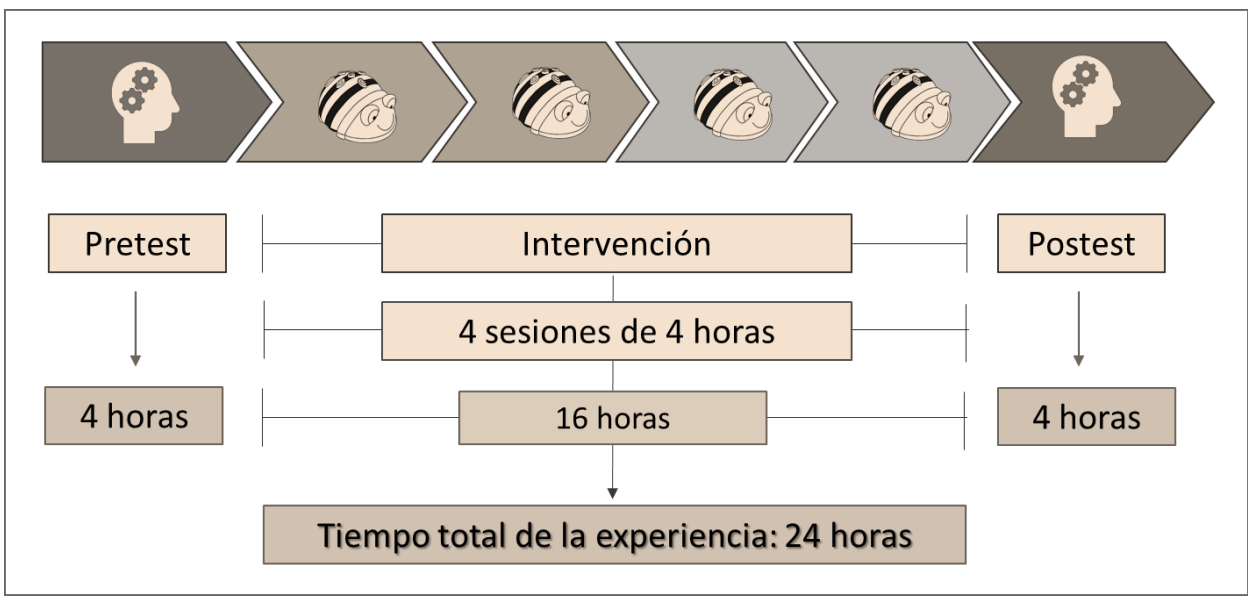

Las actividades se estructuraron en 6 sesiones. En la primera sesión se efectuó la evaluación pretest con una duración de 4 horas. Luego se desarrollaron 4 sesiones para las actividades formativas de intervención. En estas se exploró el dominio de la característica secuencia del pensamiento computacional. Cada sesión fue propuesta utilizando 4 horas en cada una de las sesiones para un tiempo total de 16 horas. Finalmente, la experiencia culminó con una sesión de evaluación postest que implicó 4 horas.

Las evaluaciones o pruebas que se realizaron (pretest y postest) se basaron en retos o desafíos de programación denominados Solve-It. Los restos consistían en la construcción de secuencias de programación para lograr que el robot Bee-Bot $囚$ se moviera hasta un punto específico marcado sobre un tapete. Cada reto propuesto iniciaba con la presentación en forma oral de una pequeña narración o historia (expuesta por el investigador) que servía como elemento motivacional para integrar al alumno en las actividades de aprendizaje (Szurmak y Mindy, 2013; Fridin, 2014). En cada evaluación se planificaron 5 retos. Los estudiantes trabajaron de forma colaborativa organizados en pequeños grupos (3-4 estudiantes) en su aula de clases cotidiana.

\section{Recursos y materiales utilizados}

En la realización de las actividades de evaluación (pretest y postest) así como para las sesiones de intervención planificadas se utilizó el robot educativo programable

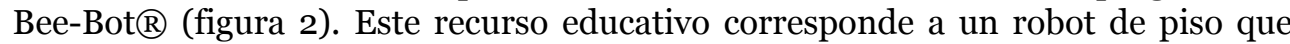
posee una forma de abeja, una característica que lo convierte en un recurso de interés para niños y niñas de primeras etapas escolares. 
Este robot puede utilizarse como interfaz tangible de aprendizaje, para introducir los conceptos de programación a los niños pequeños (García-Peñalvo et al., 2016; Gonzalez-Gonzalez, 2019). Para programar los movimientos del robot se utilizaron unos botones ubicados en su exterior. Los botones permiten indicarle al robot cuatro tipos de movimientos: adelante, atrás con un desplazamiento constante de $15 \mathrm{~cm} \mathrm{y}$ giros a la izquierda o derecha en ángulos de $90^{\circ}$.

Para el desarrollo de la experiencia educativa se diseñaron algunos tapetes que serían el escenario para el desplazamiento del robot. (figura 2).

Figura 2. Recurso de robótica educativa y tapete utilizados en la experiencia educativa

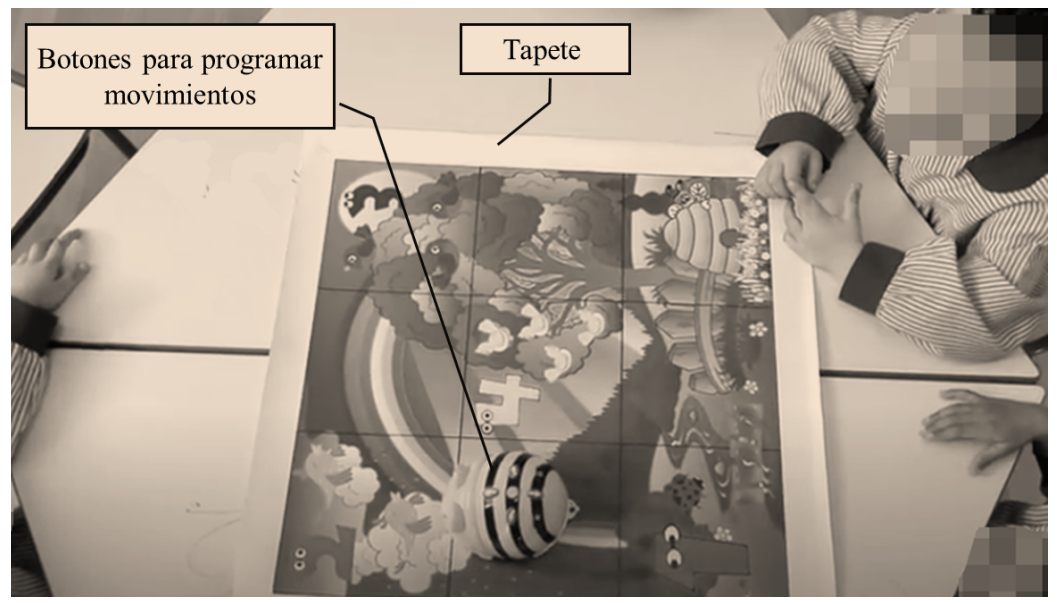

\section{Recolección de datos}

En el estudio se empleó como instrumento de recolección de datos una rúbrica. El instrumento permitió el registro del nivel de logro y dominio alcanzado por cada uno de los estudiantes en el desarrollo de los retos de programación y robótica propuestos en las evaluaciones pretest y postest. En la rúbrica era posible registrar el logro del participante de acuerdo con una escala de valores entre de o a 5 puntos.

Los criterios y valores contemplados en el instrumento fueron: el reto fue realizado totalmente y sin ayuda, con un valor de 5 puntos; el reto se efectuó parcialmente con ayuda mínima, valorado en 4 puntos; el reto fue completado con ayuda moderada y periódica, con un valor de 3 puntos; el reto fue completado recibiendo ayuda constante y paso a paso, con un valor de 2 puntos. Finalmente, si el estudiante realizaba el reto propuesto, pero no podía completarlo, el valor asignado era 1 punto. En caso de no participar del reto, no se asignaban puntos. El instrumento que fue utilizado corresponde a una adaptación de otros que se han empleado en estudios 
previos (Bers, 2010; Elkin, Sullivan y Bers, 2016; Sullivan, Bers, y Mihm, 2017; Caballero-González y García-Valcárcel, 2019; González y García-Valcárcel, 2020).

\section{ANÁLISIS Y RESULTADOS}

Para conocer el nivel de influencia que representó el desarrollo de la experiencia de aprendizaje en el dominio de la característica secuencia del pensamiento computacional se efectuó un análisis a los datos recolectados mediante la ejecución de las pruebas pre y postest, organizadas con retos de programación y robótica educativa. La característica secuencia que fue explorada en las evaluaciones corresponde a la dimensión conceptual del pensamiento computacional (Brennan y Resnick, 2012). Los datos que fueron recolectados se trataron estadísticamente mediante técnicas de tipo cuantitativas a través del programa SPSS versión 23.

$\mathrm{El}$ análisis inicial permitió comprobar la normalidad de los datos recolectados. Para esto se utilizó la prueba Kolmogorov-Smirnov (K-S), estableciendo como valor crítico un $\alpha<.05$. En la tabla 1, se presentan los valores calculados con la prueba K-S aplicado a los datos sobre el desempeño alcanzado por los estudiantes, de los grupos experimental y control, en el dominio de la característica secuencia del pensamiento computacional. Los cálculos arrojaron un valor para p (Sig.) > ,05 con lo cual se puede decir que los datos correspondientes a las evaluaciones pretest y postest siguen una distribución normal.

Tabla 1. Resultados de la prueba estadística K-S utilizando los datos del pretest y postest en los grupos experimental y control

\begin{tabular}{|l|l|c|c|c|}
\hline \multirow{2}{*}{ Grupo } & \multicolumn{4}{|c|}{ Kolmogorov-Smirnov } \\
\cline { 3 - 5 } & & Estadístico & gl & p (Sig.) \\
\hline \multirow{2}{*}{ Pretest } & Experimental &, 167 & 20 &, 147 \\
\cline { 2 - 5 } & Control &, 179 & 20 &, 093 \\
\hline \multirow{2}{*}{ Postest } & Experimental &, 178 & 20 &, 095 \\
\cline { 2 - 5 } & Control &, 182 & 20 &, 082 \\
\hline
\end{tabular}

Igualmente, a los datos de las evaluaciones pretest de ambos grupos (experimental y control) se les aplicó la prueba estadística de Levene para determinar la igualdad de varianza (tabla 2). El valor calculado para la prueba presentó un (Sig.) > ,05. Los valores permiten establecer que no existen diferencias significativas; es decir, que inicialmente los grupos son equivalentes. 
Tabla 2. Resultados de la prueba de igualdad de varianza aplicando el estadístico $t$-student a los datos del pretest en los grupos experimental y control

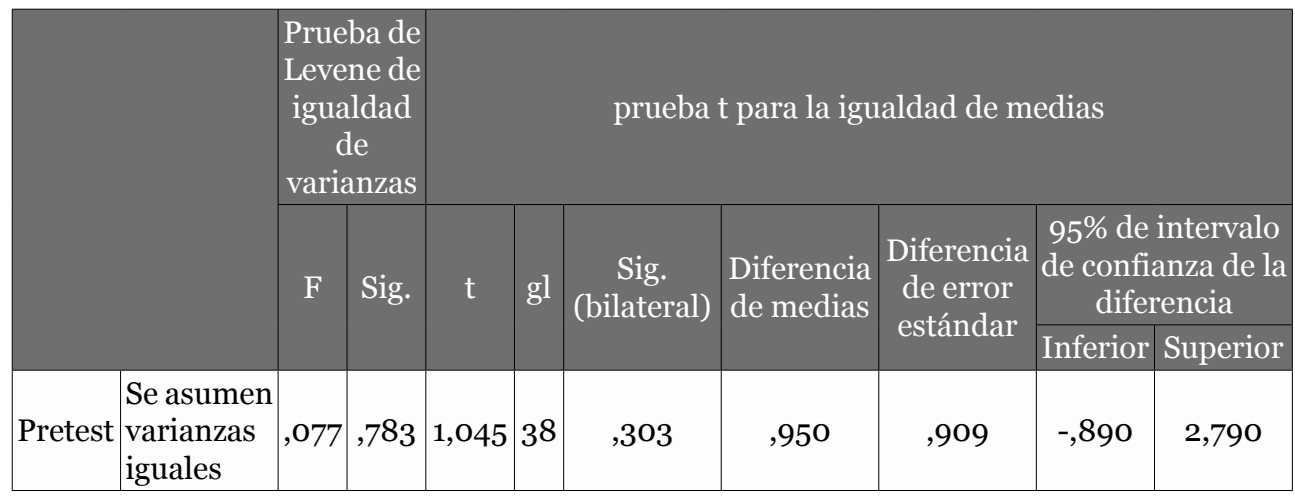

Luego de efectuar la evaluación pretest y de realizar las sesiones de intervención con actividades de formación y aprendizaje vinculadas a la característica secuencia del pensamiento computacional, se aplicó una evaluación postest. Los datos fueron recolectados mediante el mismo instrumento de recolección que se utilizó en el pretest, es decir, la rúbrica para registrar el desempeño alcanzado por los estudiantes en el desarrollo de una prueba con retos de programación y robótica educativa.

En la tabla 3 se muestran los valores de los estadísticos descriptivos que corresponden a los datos recolectados en la evaluación postest.

Tabla 3. Estadísticos descriptivos de los datos generados en la evaluación postest aplicada a los grupos experimental y control

\begin{tabular}{|l|c|c|c|c|}
\hline \multicolumn{1}{|c|}{ Grupo } & $\mathrm{N}$ & Media & $\begin{array}{c}\text { Desviación } \\
\text { estándar }\end{array}$ & $\begin{array}{c}\text { Media de error } \\
\text { estándar }\end{array}$ \\
\hline Experimental & 20 & 15,80 & 3,833 &, 857 \\
\hline Control & 20 & 13,30 & 2,922 &, 653 \\
\hline
\end{tabular}

Por otro lado, para garantizar una respuesta eficaz a la primera interrogante que se formuló para el estudio se presentan en la tabla 4 los valores generados con estadístico $t$-student aplicado a los datos provenientes de la evaluación postest en los grupos experimental y control.

Los cálculos estadísticos exponen la existencia de diferencias significativas, en la evaluación postest, a favor de los estudiantes que participaron de la actividad de formación y aprendizaje; es decir, los integrantes del grupo experimental. 
Tabla 4. Resultados del estadístico $t$-student aplicado a los datos de la evaluación postest en los grupos experimental y control

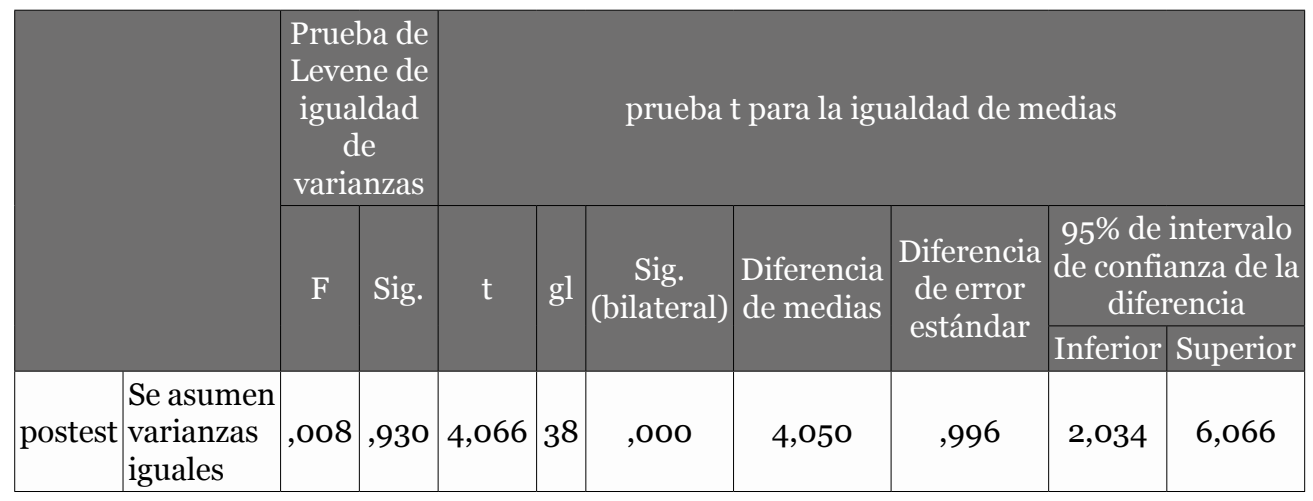

Adicionalmente, se efectuó un análisis de los datos obtenidos en la evaluación postest tomando en cuenta el sexo de los participantes. En la tabla 5 mostramos los estadísticos descriptivos que corresponden a estos datos.

Tabla 5. Estadísticos descriptivos de los datos generados en la evaluación postest considerando el sexo de los estudiantes participantes

\begin{tabular}{|c|c|c|c|c|c|}
\hline & Sexo & $\mathrm{N}$ & Media & $\begin{array}{c}\text { Desviación } \\
\text { estándar }\end{array}$ & $\begin{array}{c}\text { Media de error } \\
\text { estándar }\end{array}$ \\
\hline \multirow{2}{*}{ postest } & Niño & $\mathbf{2 2}$ & $\mathbf{1 5 , 6 4}$ & 3,472 &, 740 \\
\cline { 2 - 6 } & Niña & $\mathbf{1 8}$ & $\mathbf{1 4 , 1 7}$ & 3,959 &, 933 \\
\hline
\end{tabular}

En la tabla 6 se muestran los resultados del estadístico t-student aplicado a los datos recolectados en el postest considerando el sexo de los estudiantes. El valor calculado para la prueba presentó un $\mathrm{p}>, 05$. El resultado indica que no existen diferencias significativas en relación con el sexo de los estudiantes participantes. 
Tabla 6. Resultados del estadístico $t$-student aplicado a los datos de la evaluación postest de acuerdo con el sexo de los participantes en los grupos experimental y control

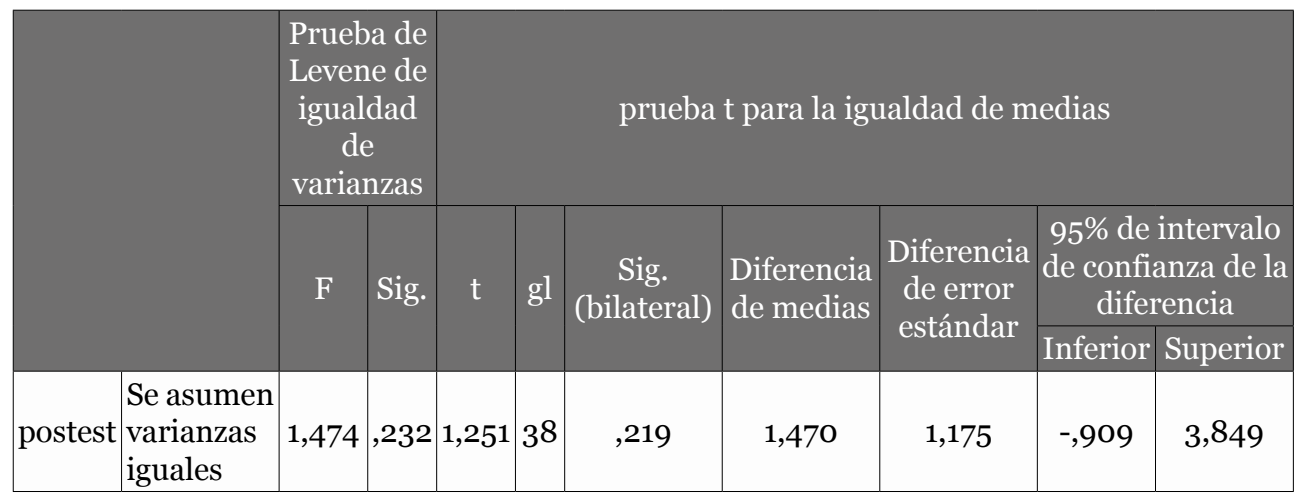

\section{DISCUSIÓN Y CONCLUSIONES}

Los resultados que se han presentado hacen referencia a los datos generados mediante el desarrollo de una experiencia educativa planificada con miras a contribuir al aprendizaje del pensamiento computacional mediante el dominio de la habilidad de secuenciar actividades/tareas/órdenes en estudiantes de educación infantil. En la experiencia educativa se estructuraron actividades basadas en retos de programación que implicaron el diseño y construcción de secuencias de movimientos aplicados al robot educativo Bee-Bot $\AA$.

Los resultados de las evaluaciones pre/postest permitieron responder a las interrogantes formuladas para el estudio. Igualmente, fue posible comprobar la hipótesis de partida donde se argumentaba que los estudiantes que participaban de una experiencia de formación y aprendizaje lograban un dominio y aprendizaje de habilidades vinculadas a la programación y el pensamiento computacional.

En este sentido, en relación con la primera interrogante, los valores de los cálculos realizados exponen la existencia de diferencias significativas entre los estudiantes. Las diferencias estaban asociadas al grupo en el cual participaron. Es decir, que los resultados favorecían a los participantes del grupo experimental. Los estudiantes de este grupo manifestaron un desempeño o logro mayor en la secuenciación de movimientos del robot a los obtenidos por los integrantes del grupo control, que no participaron de las actividades de formación y aprendizaje.

Por otro lado, como respuesta a la segunda interrogante se pudo establecer que mediante los análisis aplicados a los valores de logro o desempeño recolectados y comparando la muestra en función del sexo de los estudiantes que participaron en el programa formativo, no se produjeron diferencias estadísticamente significativas. Por lo cual se puede afirmar que se produjo un desarrollo similar de esta habilidad 
entre niñas y niños de primeras etapas educativas; es decir, que las actividades de aprendizaje propuestas permiten romper con los estereotipos de género que sustentan una mayor prevalencia del nivel de logro en participantes de sexo masculino.

En consecuencia, podemos sostener que los resultados obtenidos guardan relación con otros estudios que se han efectuado previamente y donde igualmente se proponen los beneficios y posibilidades de fortalecer el dominio del pensamiento computacional desde la infancia temprana, utilizando robots programables como interface tangible de aprendizaje (Ramírez y Sosa, 2013; Sullivan y Bers, 2013; Di Lieto et al., 2017; Sullivan, Strawhacker y Bers, 2017; Chalmers, 2018; Karampinis, 2018; García-Peñalvo y Mendes, 2018; Santoya-Mendoza et al., 2018; Goodgame, 2018; Horn y Bers, 2019; Sullivan y Bers, 2019).

Como limitantes del estudio podemos señalar: el espacio físico de trabajo, la cantidad de kits de robótica educativa utilizados y el tamaño de la muestra. En la planificación de nuevos estudios vinculados a esta línea de investigación sería conveniente considerar estos y otros aspectos como la exploración de más dimensiones del pensamiento computacional, diversificar el nivel educativo y lograr una participación más activa de los padres de familia. Sin embargo, este trabajo se puede considerar como un apoyo significativo al campo de conocimiento existente en relación con el fortalecimiento de habilidades digitales vinculadas al aprendizaje y dominio de la programación y el pensamiento computacional en las primeras etapas escolares.

\section{REFERENCIAS}

Angeli, C., Voogt, J., Fluck, A., Webb, M., Cox, M., Malyn-Smith, J., y Zagami, J. (2016). A K-6 computational thinking curriculum framework: Implications for teacher knowledge. Journal of Educational Technology \& Society, 19(3). www.jstor. org/stable/jeductechsoci.19.3.47

Berrocoso, J., Sánchez, M., y Arroyo, M. (2015). El pensamiento computacional y las nuevas ecologías del aprendizaje. Red, 46, 1-18. https://doi.org/10.6018/ $\mathrm{red} / 46 / 3$

Bers, M. U. (2008). Blocks, robots and computers: Learning about technology in early childhood. New York: Teacher's College Press.

Bers, M. U. (2010). The TangibleK Robotics program: Applied computational thinking for Young children. Early Childhood Research \& Practice, 12(2). https://bit. ly/2RZ3B11
Bers, M. U. (2012). Designing Digital Experiences for Positive Youth Development: From Playpen to Playground. Oxford: University Press. https://doi.org/10.1093/ acprof:0so/9780199757022.001.0001

Bers, M. U. (2017). The Seymour test: Powerful ideas in early childhood education. International Journal of Child - Computer Interaction, 14, 10-14. https:// doi.org/10.1016/j.ijcci.2017.06.004

Bers, M.U.(2018). Coding and Computational Thinking in Early Childhood: The Impact of Scratch Jr in Europe. European Journal of STEM Education, 3(3), o8. https://doi. org $/ 10.20897 /$ ejsteme $/ 3868$

Bers, M. U., Flannery, L., Kazakoff, E. R., y Sullivan, A. (2014). Computational thinking and tinke-ring: Exploration of an early childhood robotics curriculum. Computers \& Education, 
72, 145-157. https://doi.org/10.1016/j. compedu.2013.10.020

Brennan, K., y Resnick, M. (2012). New frameworks for studying and assessing the development of computational thinking. In Proceedings of the 2012 Annual Meeting of the American Educational Research Association (AERA) (pp. 1-25), Vancouver, Canada.

Bruni, F., y Nisdeo, M. (2017). Educational robots and children's imagery: A preliminary investigation in the first year of primary school. Research on Education and Media, 9(1), 37-44. https://doi. org/10.1515/rem-2017-0007

Caballero-González, Y. A., y García-Valcárcel, A. (2019). Fortaleciendo habilidades de pensamiento computacional en Educación Infantil: Experiencia de aprendizaje mediante interfaces tangible y gráfica. Revista Latinoamericana de Tecnología Educativa-RELATEC, 18(2). https://doi. org/10.17398/1695-288X.18.2.133

Campbell, D., y Stanley, J. (1993). Diseños experimentales y cuasiexperimentales en la investigación social. Amorrortu.

Cejka, E., Rogers, C., y Portsmore, M. (2006). Kindergarten robotics: Using Robotics to motivate math, science, and engineering literacy in elementary school. International Journal of Engineering Education, 22(4), 711-722.

Chalmers, C. (2018). International Journal of Child-Computer Interaction Robotics and computational thinking in primary school. International Journal of Child-Computer Interaction, 17, 93-100. https://doi. org/10.1016/j.ijcci.2018.06.005

Cheng, Y. W., Sun, P. C., y Chen, N. S. (2018). The essential applications of educational robot: Requirement analysis from the perspectives of experts, researchers and instructors. Computers \& education, 126, 399-416. https://doi.org/10.1016/j. compedu.2018.07.020

Di Lieto, M. C., Inguaggiato, E., Castro, E., Cecchi, F., Cioni, G., Dell'Omo,
M., ... y Dario, P. (2017). Educational Robotics intervention on Executive Functions in preschool children: A pilot study. Computers in Human Behavior, 71, 16-23. https://doi.org/10.1016/j. chb.2017.01.018

Elkin, M., Sullivan, A., y Bers, M. U. (2016). Programming with the KIBO robotics kit in preschool classrooms. Computers in the Schools, 33(3), 169-186. https://doi. org/10.1080/07380569.2016.1216251

Fridin, M. (2014). Storytelling by a kindergarten social assistive robot: A tool for constructive learning in preschool education. Computers \& education, 70, 53-64. https://doi.org/10.1016/j. compedu.2013.07.043

García-Peñalvo, F. J., Rees, A. M., Hughes, J., Jormanainen, I., Toivonen, T., y Vermeersch, J. (2016). A survey of resources for introducing coding into schools. Proceedings of the Fourth Inter-national Conference on Technological Ecosystems for Enhancing Multiculturality, TEEM'16. (pp.1926). Salamanca, Spain, November 2-4, 2016. New York: ACM. https://doi. org $/ 10.1145 / 3012430.3012491$

García-Peñalvo, F. J., y Mendes, A. J. (2018). Exploring the computational thinking effects in pre-university education. Computers in Human Behavior, 80, 407-411. https://doi.org/10.1016/j. chb.2017.12.005

García-Valcárcel, A., y Hernández, A. (2013). Recursos tecnológicos para la enseñanza e innovación educativa. Editorial Síntesis Goodgame, C. (2018). Beebots and Tiny Tots. In E. Langran, y J. Borup (Eds.), Society for Information Technology \& Teacher Education International Conference (1179-1183). Association for the Advancement of Computing in Education (AACE).

González-González, C. S. (2019). Estrategias para la enseñanza del pensamiento computacional y uso efectivo de 
tecnologías en educación infantil: una propuesta inclusiva. RIITE. Revista Interuniversitaria de Investigación en Tecnología Educativa, 7, 85-97. https:// doi.org/10.6018/riite.405171

González-González, C. S. (2019). State of the art in the teaching of computational thinking and pro-gramming in childhood education. Education in the Knowledge Society, 20, 17. https://doi.org/10.14201/ eks2019 $20 \quad$ a17

González-Martínez, J., Estebanell-Minguell, M., y Peracaula-Bosch, M. (2018). ¿Robots o programación? El concepto de Pensamiento Computacional y los futuros maestros. Education in the Knowledge Society (EKS). https://doi.org/10.14201/ eks20181922945

González, Y. A. C., y García-Valcárcel, A. (2017, November). Educational robotics for the formation of programming skills and computational thinking in childish. In 2017 International Symposium on Computers in Education (SIIE) (pp. 1-5). IEEE. https://doi.org/10.1109/ SIIE.2017.8259652

González, Y. A. C., y García-Valcárcel, A. (2018, October). A robotics-based approach to foster programming skills and computational thinking: Pilot experience in the classroom of early childhood education. In Proceedings of the Sixth International Conference on Technological Ecosystems for Enhancing Multiculturality (pp.41-45),ACM.https:// doi.org/10.1145/3284179.3284188

González, Y. A. C., y García-Valcárcel, A. (2020). ¿Aprender con robótica en Educación Primaria? Un medio de estimular el pensamiento computacional. Education in the knowledge society (EKS), 21(10). https://doi.org/10.14201/ eks.22957

Grover, S., y Pea, R. (2013). Computational Thinking in K-12: A Review of the State of the Field. Educational
Researcher, 42(1), 38-43. https://doi. org/10.3102/0013189X12463051

Henriksen, D., Henderson, M., Creely, E, Ceretkova, S., Černochová, M., Sendova, E., Sointu, E. T., y Tienken, C. T. (2018). Creativity and Technology in Education: An International Perspective. Technology, Knowledge and Learning, 23, 409-424. https://doi.org/10.1007/s10758-0189380-1

Hernández Sampieri, R., FernándezCollado, C., y Baptista-Lucio. P. (2014). Metodología de la in-vestigación. McGraw-Hill Education.

Horn, M., y Bers, M. (2019). Tangible Computing. In The Cambridge Handbook of Computing Edu-cation Research (S.A. Fincher and A.V. Robins, Eds.), Cambridge University Press. https://doi. org/10.1017/9781108654555.023

Kalelioğlu, F. (2015). A new way of teaching programming skills to K-12 students: Code.org. Computers in Human Behavior, 52, 200-210. https://doi. org/10.1016/j.chb.2015.05.047

Karampinis, T. (2018). Robotics-based learning interventions and experiences from our implementations in the RobESL framework. International Journal of Smart Education and Urban Society, 9(1), 13-24. https://doi.org/10.4018/ IJSEUS.2018010102

Kucuk, S., y Sisman, B. (2017). Behavioral patterns of elementary students and teachers in one-to-one Robotics instruction. Computers \& Education, 111, 31-43. https://doi.org/10.1016/j. compedu.2017.04.002

Lee, I., Martin, F., y Apone, K. (2014). Integrating computational thinking across the $\mathrm{K}-8$ curriculum. $A C M$ Inroad, 5(4), 64-71. https://doi. org/10.1145/2684721.2684736

Liu H. P., Perera S. M., y Klein J. W. (2017) Using Model-Based Learning to Promote Computational Thinking Education. In P. Rich y C. Hodges (Eds.), Emerging 
Research, Practice, and Policy on Computational Thinking. Educational Communications and Technology: Issues and Innovations. Springer, Cham. https://doi.org/10.1007/978-3-31952691-1 10

Metz, S. S. (2007). Attracting the engineering of 2020 today. In R. Burke y M. Mattis (Eds.), Women and minorities in science, technology, engineering and mathematics: Upping the numbers (184209). Edward Elgar Publishing.

Merrill, M. D. (2009). First principles of instruction. In C. M. Reigeluth y A. A. Carr-Chellman (Eds.), Instructionaldesign theories and models: Building a common knowledge base (Vol. III), (4156). Routledge.

Papert, S. (1980). Mindstorms: Children, computers, and powerful ideas. Basic Books.

Papadakis, S., Kalogiannakis, M., y Zaranis, N. (2016). Developing fundamental programming concepts and computational thinking with ScratchJr in preschool education: a case study. International Journal of Mobile Learning and Organization, 1O(3), 187. https://doi. org/10.1504/IJMLO.2016.077867

Ramírez, P. A. L., y Sosa, H. A. (2013). Aprendizaje de y con robótica, algunas experiencias. Revista Educación, 37(1), 43-63. https://doi.org/10.15517/revedu. v37i1.10628

Reigeluth, C. M. (2016). Teoría instruccional y tecnología para el nuevo paradigma de la educación. RED. Revista de Educación a Distancia, 50. https://doi.org/10.6018/ red/50/1a

Relkin, E., y Bers, M. U. (2019). Designing an assessment of computational thinking abilities for young children. STEM for early childhood learners: how science, technology, engineering and mathematics strengthen learning. Routledge. https:// doi.org/10.4324/9780429453755-5
Resnick, M., y Rosenbaum, E. (2013). Designing for tinkerability. Design, Make, Play: Growing the Next Generation of STEM Innovators, 163-181. https://doi. org/10.4324/9780203108352

Santoya-Mendoza, A., Díaz-Mercado, A., Fontalvo-Caballero, F., Daza-Torres, L., Avendaño-Bermúdez, L., SánchezNoriega, L., Ramos-Bernal, P., BarriosMartínez, E., López-Daza, M., OsorioCervantes, G., Rodríguez-Pertuz, M., y Moreno-Polo, V. (2018). Robótica educativa desde la investigación como estrategia pedagógica apoyada en TIC en la escuela. Cultura. Educación y Sociedad 9(3), 699-708. https://doi.org/10.17981/ cultedusoc.9.3.2018.82

Selby, C., y Woollard, J. (2013). Computational thinking: the developing definition. https://cutt.ly/vryITzu

Siu-Cheung, K. (2019). Components and Methods of Evaluating Computational Thinking for Fostering Creative Problem-Solvers in Senior Primary School Education. Computational thinking Education. NY: Springer Berlin Heidelberg. https://doi.org/10.1007/978981-13-6528-7 8

Strawhacker, A., y Bers, M. U. (2015). "I want my robot to look for food": Comparing Kindergartner's programming comprehension using tangible, graphic, and hybrid user interfaces. International Journal of Technology and Design Education, 25(3), 293-319. https://doi. org/10.1007/s10798-014-9287-7

Strawhacker, A., y Bers, M. U. (2019). Promoting Positive Technological Development in a Kinder-garten Makerspace: A Qualitative Case Study. European Journal of STEM Education, 3(3), 09. https://doi.org/10.20897/ ejsteme/3869

Sullivan, A., y Bers, M. U. (2013). Gender differences in kindergarteners' robotics and programming achievement. International journal of technology and 
designeducation, 23(3), 691-702. https:// doi.org/10.1007/s10798-012-9210-Z

Sullivan, A., y Bers, M. U. (2016). Robotics in the early childhood classroom: learning outcomes from an 8-week robotics curriculum in pre-kindergarten through second grade. International Journal of Technology and Design Education, 26(1), 3-20. https://doi.org/10.1007/s10798015-9304-5

Sullivan, A., y Bers, M. U. (2018). Dancing robots: integrating art, music, and robotics in Singapo-re's early childhood centers. International Journal of Technology and Design Education, 28(2), 325-346. https://doi.org/10.1007/s10798-0179397-0

Sullivan, A., y Bers, M. U. (2019). Investigating the use of robotics to increase girls' interest in engineering during early elementary school. International Journal of Technology and Design Education, 29(5), 1033-1051. https://doi. org/10.1007/s10798-018-9483-y

Sullivan, A., Bers, M. U., y Mihm, C. (2017). Imagining, playing, and coding with KIBO: using robotics to foster computational thinking in young children. Siu-cheung KONG The Education University of Hong Kong, Hong Kong, 110.
Sullivan, A., Strawhacker, A., y Bers, M. U. (2017). Dancing, Drawing, and Dramatic Robots: Inte-grating Robotics and the Arts to Teach Foundational STEAM Concepts to Young Children. En M. S. Khine (Ed.), Robotics in STEM Education: Redesigning the Learning Experience (231-260). Springer International Publishing. https://doi.org/10.1007/9783-319-57786-9 10

Szurmak, J., y Mindy, T. (2013). Tell me a story: The use of narrative as a tool for instruction. In Imagine, Innovate, Inspire: The Proceedings of the ACRL 2013 Conference (pp. 546-552). Indianapolis, IN, USA: ACRL.

Vee, A. (2013). Understanding computer programming as a literacy. Literacy in Composition Studies, 1(2), 42-64. https:// doi.org/10.21623/1.1.2.4

Wing, J. M. (2006). Computational thinking. Communications of the ACM, 49(3),33-35. https://doi.org/10.1145/1118178.1118215

Zapata-Ros, M. (2015). Pensamiento computacional: Una nueva alfabetización digital. Red, 46, 1-47. https://doi. org $/ 10.6018 / \mathrm{red} / 45 / 4$

Zapata-Ros, M. (2019). Computational Thinking Unplugged. Education in the Knowledge Society, 20, 18. https://doi. org/10.14201/eks2019 20 a18

\section{PERFIL ACADÉMICO Y PROFESIONAL DE LOS AUTORES}

Yen-Air Caballero-González. Participó como miembro del Grupo de Investigación-Innovación en Tecnología Educativa GITE-USAL de la Universidad de Salamanca como doctorando del programa Formación en la Sociedad del Conocimiento. Sus líneas de investigación están asociadas a la integración de las TIC en los procesos educativos, su tesis doctoral hace referencia al aprendizaje del Pensamiento Computacional mediante escenarios de aprendizaje con robótica educativa. Además, es Profesor Colaborador del Grupo GITCE de la Universidad Tecnológica de Panamá. https://orcid.org/0000-0002-7493-6683

E-mail: ycaballero@usal.es 
Ana García-Valcárcel Muñoz-Repiso. Catedrática del Departamento de Didáctica, Organización y Métodos de Investigación de la Universidad de Salamanca. Participa de la Red Universitaria de Tecnología Educativa (RUTE), la Asociación de Tecnología Educativa (EDUTEC) y la Red de Innovación Educativa (REUNI+D). $\mathrm{Su}$ investigación se ha focalizado en diferentes niveles educativos, explorando la incidencia del desarrollo de las TIC en los procesos de enseñanza-aprendizaje, la formación del profesorado y la evaluación de las competencias digitales de los estudiantes. https://orcid.org/0000-0003-0463-0192

E-mail: anagv@usal.es

Dirección:

Facultad de Educación

Universidad de Salamanca

Paseo de Canalejas, 169

37008, Salamanca

Fecha de recepción del artículo: 14/05/2020

Fecha de aceptación del artículo: 02/09/2020

Fecha de aprobación para maquetación: 17/09/2020 